\section{Morfometría litiásica como predictor del número de accesos o procedimientos en nefrolitotomía percutánea}

Sedano-Basilio JE, Trujillo-Ortiz L, Herrera-Muñoz JA, Gómez-Sánchez J, Preciado-Estrella DA, López-Maguey RP, Sánchez-Aquino UC, Calvo-Vázquez I, Cortés-Raygoza PA, Hernández-Méndez EA, Ortega-González ME, MartínezArroyo C, Cantellano-Orozco M, Morales-Montor JG, Pacheco-Gahbler C

\section{Resumen}

ANTECEDENTES: no existe consenso acerca del término "litiasis coraliforme", las designaciones "parcial" o "completa" no implican criterios de volumen. El término "morfometría litiásica" se define como la distribución de la carga volumétrica de un lito coraliforme en el sistema colector.

OBJETIVO: determinar la "morfometría litiásica" mediante un estudio preoperatorio de tomografía.

MATERIALES Y MÉTODOS: ensayo retrospectivo-analítico al que se incluyeron pacientes a quienes entre los años 2012-2016 se les realizó un estudio tomográfico. Para la medición de los volúmenes: litiásico total (volumen litiásico total : volumen calculado con reconstrucción de toda la imagen del lito), litiásico del cáliz de entrada (VLCE: volumen del componente en cáliz de entrada), litiásico de cáliz favorable (litiásico de cáliz favorable: volumen del componente en cáliz favorable) se utilizó el programa de cómputo OsiriX.

RESULTADOS: se incluyeron 46 pacientes en quienes se observaron, volumen calculado con reconstrucción de toda la imagen del lito: $2753 \pm 1423 \mathrm{~mm}^{3}$ y $56867 \pm 26781 \mathrm{~mm}^{3}(\mathrm{p}<0.001)$; volumen del componente en cáliz de entrada: $580 \pm 474 \mathrm{~mm}^{3}$ y $17586 \pm 13150$ $\mathrm{mm}^{3}$ ( $\left.\mathrm{p}<0.001\right)$; volumen del componente en cáliz favorable: $589 \pm$ $569 \mathrm{~mm}^{3}$ y $17900 \pm 13717 \mathrm{~mm}^{3}(\mathrm{p}<0.001)$ en casos con tracto único y más de 2 , respectivamente.

CONCLUSIONES: la morfometría litiásica proporciona la carga volumétrica del lito a tratar e influye significativamente en la determinación de los tractos o procedimientos necesarios para alcanzar un estado libre de litiasis en pacientes con litos coraliformes a quienes se realiza nefrolitotomía percutánea en nuestra población.

PALABRAS CLAVE: litiasis, coraliforme, morfometría litiásica, tracto, procedimiento, nefrolitotomía percutánea.
División de Urología, Hospital General Dr. Manuel Gea González, Secretaría de Salud, Ciudad de México.

Recibido: diciembre 2016

Aceptado: marzo 2017

Correspondencia

Jorge E Sedano Basilio

drjsedanob@gmail.com

Este artículo debe citarse como

Sedano-Basilio JE, Trujillo-Ortiz L, Herrera-Muñoz JA, Gómez-Sánchez J, Preciado-Estrella DA, LópezMaguey RP, Sánchez-Aquino UC, Calvo-Vázquez I, Cortés-Raygoza PA, Hernández-Méndez EA, Ortega-González ME, Martínez-Arroyo C, CantellanoOrozco M, Morales-Montor JG, Pacheco-Gahbler C. Morfometría litiásica como predictor del número de accesos o procedimientos en nefrolitotomía percutánea. Rev Mex Urol. 2017 mar;77(2):125-137. 


\section{Stone morphometry: tract and stage predictor for percutaneous nephrolithotomy}

Sedano-Basilio JE, Trujillo-Ortiz L, Herrera-Muñoz JA, Gómez-Sánchez J, Preciado-Estrella DA, López-Maguey RP, Sánchez-Aquino UC, Calvo-Vázquez I, Cortés-Raygoza PA, Hernández-Méndez EA, Ortega-González ME, MartínezArroyo C, Cantellano-Orozco M, Morales-Montor JG, Pacheco-Gahbler C

\section{Abstract}

BACKGROUND: there is no consensus on the term "staghorn stone" and the designations "partial" or "complete" do not involve volume criteria. The term "staghorn morphometry" is defined as the distribution of the volumetric burden of a staghorn stone in the collecting system.

OBJECTIVE: to determine the "stone morphometry" through preoperative tomography study.

MATERIALS AND METHODS: a retrospective, analytic study was conducted that included patients with tomography studies within the time frame of 2012-2016. OsiriX software was used with measurements of: total stone volume (volume calculated with reconstruction of the entire stone image), stone volume in the entry calyx (volume of the stone in the entry calyx), stone volume in the favorable calyx (volume of the stone in the favorable calyx).

RESULTS: the following overall measurements were observed in the 46 patients included in the study: total stone volume: $2753 \pm 1423$ $\mathrm{mm}^{3}$ and $56867 \pm 26781 \mathrm{~mm}^{3}(\mathrm{p}<0.001)$, stone volume in the entry calyx: $580 \pm 474 \mathrm{~mm}^{3}$ and $17586 \pm 13150 \mathrm{~mm}^{3}(\mathrm{p}<0.001)$, and stone volume in the favorable calyx: $589 \pm 569 \mathrm{~mm}^{3}$ and $17900 \pm 13717$ $\mathrm{mm}^{3}(\mathrm{p}<0.001)$, in cases with a single tract and in those with more than 2 tracts, respectively.

CONCLUSIONS: stone morphometry provided the volumetric load of the stone to be treated and was significantly influential in determining the tracts and procedures necessary to achieve a stone-free state in patients with staghorn stones that underwent percutaneous nephrolithotomy in a Mexican hospital population.

KEY WORDS: lithiasis, staghorn, stone morphometry, tract, procedure, percutaneous nephrolithotomy
División de Urología, Hospital General Dr. Manuel Gea González, Secretaría de Salud, Ciudad de México.

Correspondence

Jorge E Sedano Basilio drjsedanob@gmail.com

\section{ANTECEDENTES}

La litiasis urinaria, por su prevalencia, es un problema de salud relevante $(2-3 \%$ de la población en general) y, más aún, porque su mayor incidencia es en la población activa (30 a 50 años)..$^{1,2}$ Se describen como factores de riesgo: hipercalciuria, hiperuricosuria, 
volumen urinario, dieta y factores genéticos, entre otros. ${ }^{3,4}$

Hasta el decenio de 1980 el tratamiento de la litiasis renal requería intervenciones quirúrgicas mayores, en ocasiones con elevada morbilidad perioperatoria y alto riesgo de pérdida de la unidad renal tratada. El desarrollo de nuevas técnicas quirúrgicas, como la nefrolitotomía percutánea (Fernström y Johansson, 1976) y la litotricia extracorpórea por ondas de choque (LEOCH) (Chaussy, 1980) revolucionaron el enfoque terapéutico, aportando alternativas de menor grado de invasión. ${ }^{5}$

Las indicaciones de la nefrolitotomía percutánea son: cálculos renales radio-opacos (nivel de evidencia [NE]: 1b; grado de recomendación [GR]: A), cálculos renales de cistina (NE: 2a, GR: B), litiasis renal mayor de $20 \mathrm{~mm}$ (NE: 2a, GR: B), litos renales menores de $20 \mathrm{~mm}$ localizados en el polo inferior, en pacientes no aptos para litotricia extracorpórea por ondas de choque o cirugía intrarrenal endoscópica retrógrada (CIER). ${ }^{6,7,8}$

En la planificación de una punción para nefrolitotomía percutánea deben tomarse en cuenta los detalles anatómicos extrarrenales importantes: ubicación retroperitoneal del riñón, su relación con el diafragma, pleura, hígado, bazo, colon y el sitio de entrada en la piel. La fluoroscopia es la modalidad de imagen más utilizada durante el procedimiento, con el inconveniente de la exposición a la radiación ionizante, donde podría ser de utilidad el ultrasonido, que tiene la ventaja de identificar estructuras adyacentes, pero es necesaria una curva de aprendizaje..$^{9,10}$

La técnica de la punción calicial es primordial porque de ella depende el éxito o fracaso del procedimiento. Es necesario determinar con precisión el cáliz de entrada ideal para acceder al cálculo, pues la posición del riñón y su configuración obligan, a veces, a entrar en el riñón por un cáliz que no es, teóricamente, el mejor situado. ${ }^{10}$

En el último decenio la nefrolitotomía percutánea se ha utilizado como tratamiento de elección para la litiasis coraliforme. Desafortunadamente, no existe consenso para precisar la definición del término "litiasis coraliforme". Por lo tanto, el término "coraliforme" se aplica para definir cualquier lito con extensiones que ocupa más de una porción del sistema colector. La designación de litiasis coraliforme "parcial" o "completa" no implica ningún criterio específico de volumen. Las tasas de "libre de litiasis" posteriores a nefrolitotomía percutánea con monoterapia o terapia "emparedado" son sumamente dependientes de la carga litiásica. Más importante que la carga litiásica es la "morfometría litiásica o coralifor$\mathrm{me}^{\prime \prime}$, que se define como la distribución de la carga volumétrica de un lito coraliforme en el sistema colector. ${ }^{11,12,13}$

La urotomografía con reconstrucción tridimensional es una herramienta adicional para caracterizar al lito coraliforme antes de la intervención y correlacionar su significación terapéutica con la nefrolitotomía percutánea como monoterapia en términos de número de tractos y/o número de procedimientos necesarios para obtener un estado libre de litiasis. Además proporciona un nuevo armamento para la definición clínica de la litiasis coraliforme en una clasificación operatoria mas relevante para la planeación de la nefrolitotomía percutánea como monoterapia. ${ }^{12,13}$

Con base en hallazgos radiológicos Thomas y colaboradores desarrollaron la puntuación de Guy-Stone para evaluar el grado de complejidad de una nefrolitotomía percutánea. Existen otros métodos para este tipo de evaluación, como la nefrolitometría de STONE y el nomograma nefrolitométrico de CROES. Para valorar la complejidad del procedimiento estos modelos, 
además de proporcionar las características radiológicas del cálculo, toman en cuenta otros rasgos de los pacientes, como: obesidad, antecedentes quirúrgicos renales, lesión medular, espina bífida, y la experiencia del cirujano. Aunque tales valoraciones proporcionan información con respecto de la complejidad, no aportan al urólogo un estimado del riesgo que puede utilizarse para las decisiones clínicas con respecto al número de procedimientos, ni tractos a realizar. ${ }^{14,15}$

La meta de la nefrolitotomía percutánea es asegurar el completo aclaramiento de litiasis con la mínima morbilidad. Los nuevos desarrollos en estudios de imagen, técnicas e instrumentación son decisivos para conseguir estos objetivos. Diversos estudios han demostrado que con la nefrolitotomía percutánea no existe riesgo de daño a la función renal, incluidos los múltiples tractos o procedimientos. ${ }^{16,17,18}$ En 2016 Gorbachinsky y colaboradores reportaron que en los pacientes con múltiples tractos se reduce la función renal versus los pacientes con un único tracto. ${ }^{19}$

La morfometría litiásica es una nueva herramienta pronóstica para predecir la posición de los tractos y procedimientos necesarios para el tratamiento de pacientes con litiasis coraliforme mediante nefrolitotomía percutánea. La morfometría litiásica propone un nuevo sistema de clasificación para la litiasis coraliforme que la categoriza en tres tipos diferentes, dependiendo del volumen litiásico y su distribución en el sistema colector: Tipo 1: litos coraliformes con volumen litiásico total menor de $5000 \mathrm{~mm}^{3}$ y volumen litiásico porcentual en cáliz no favorable menor de 5\%. Tipo 2: volumen litiásico total $5000-20,000 \mathrm{~mm}^{3}$ y porcentaje de volumen litiásico en cáliz no favorable entre 5 y $10 \%$. Tipo 3: litos coraliformes con volumen litiásico total mayor de 20,000 $\mathrm{mm}^{3}$ y un volumen litiásico porcentual en cáliz no favorable mayor de $10 \%$. Con base en modelos estadísticos para lograr tasas libres de litiasis con monoterapia, el Tipo
1 requerirá solo un procedimiento y un tracto, el Tipo 2 un tracto y uno o más procedimientos, o múltiples tractos en un procedimiento, y el Tipo 3, por tanto, requerirá múltiples tractos y procedimientos. $^{20}$

El objetivo de este ensayo consiste en determinar el volumen, distribución y morfometría litiásicas y la "morfometría litiásica" mediante un estudio preoperatorio de tomografía.

\section{MATERIALES Y MÉTODOS}

Análisis retrospectivo llevado a cabo entre enero de 2012 y junio de 2016 de unidades renales a las que se realizó nefrolitotomía percutánea con litiasis renal mayor de $20 \mathrm{~mm}$, con tasas posoperatorias libres de litiasis. Estudio efectuado en la División de Urología del Hospital Dr. Manuel Gea González de la Ciudad de México. Criterio de exclusión: pacientes con anatomía anómala (riñón en herradura, riñones ectópicos o malrotados, pelvis bífida, uréteres bífidos) o menores de 13 años. Se trataron con tracto único o múltiples, en uno o varios procedimientos, según fuera el caso, para alcanzar un estado libre de litiasis. Antes del procedimiento quirúrgico se efectuaron exámenes de laboratorio: biometría hemática completa, química sanguínea, pruebas de coagulación, examen general de orina y urocultivo. Los pacientes con infección de vías urinarias se trataron con antibiótico, previo al procedimiento quirúrgico.

En todos los casos el estudio de gabinete fue la urotomografía, con tomógrafo espiral, con cortes de espesor de $2 \mathrm{~mm}$ (Somaton Sensation $64^{\mathrm{R}}$ CT Scanner, Siemens Healthcare, Erlangen, Germany), que se tomó antes del tratamiento quirúrgico definitivo y, subsecuentemente, se Ilevó a cabo la nefrolitotomía percutánea por un urólogo. En cada procedimiento se intentó el menor número de tractos, necesarios para alcanzar un estado libre de litiasis, con un nefroscopio y 
cistoscopio. En lo posible, se limitó el tiempo de posición en decúbito prono o irrigación hasta un límite de 120 minutos, como protocolo de nuestro hospital. El estado libre de litiasis se corroboró a los tres meses de posoperatorio.

El estado libre de carga litiásica se observó, intraoperatoriamente, con visión directa mediante nefroscopia y, en el posoperatorio, con estudios de gabinete. A todos los pacientes se les tomó una radiografía simple de abdomen, en posición antero-posterior, y una tomografía simple de abdomen (a los tres meses de posoperatorio, en búsqueda de litiasis residual). En los pacientes en quienes se documentaron fragmentos residuales ( $\geq 5 \mathrm{~mm}$ de diámetro mayor) se efectuaron los procedimientos necesarios para alcanzar una tasa libre de litiasis (eliminación completa de la litiasis demostrada durante el transoperatorio con visión directa con nefroscopia o, en el posoperatorio a los tres meses con una tomografía simple de abdomen, con fragmentos litiásicos $\leq$ $4 \mathrm{~mm}$ de diámetro mayor).

El programa de cómputo visor de imágenes OsiriX ${ }^{R}$ v.7.5.1 (Pixmeo SARL company, Bernex, Switzerland), es una aplicación útil para la interpretación, procesamiento y análisis de archivos tipo DICOM (digital imaging and communications in medicine). Este programa tiene diferentes tipos de visualización de estudios tomográficos en planos: axial, coronal, sagital e incluso logra reconstrucciones volumétricas en modelos de superficie en 3D, con obtención de las medidas necesarias a partir de la selección de las distintas regiones de interés (ROI). El cálculo del volumen se basa en la geometría de la superficie de las zonas de estudio y en los parámetros calibrados en el programa de cómputo.

\section{Análisis de la urotomografía}

Los estudios radiológicos pre y posoperatorios se efectuaron con el programa procesador de imágenes Synapse ${ }^{R}$ PACS (Fujifilm Medical
Systems, USA, Inc., Stamford, CT) con reconstrucción en cortes axiales, coronales, sagitales y en 3D con el programa visor de imágenes OsiriX ${ }^{R}$ v.7.5.1 (Pixmeo SARL company, Bernex, Swtizerland), con medición de los siguientes parámetros: número, localización y densidad de litos (Unidades Hounsfield), carga litiásica (Stone Burden $=0.785 \times$ Longitud $_{\text {Max }} \times$ Ancho $\left.{ }_{\max }\right)$, volumen litiásico total (VLT): volumen calculado con la reconstrucción de toda la imagen del lito; volumen litiásico pélvico (VLP): volumen calculado mediante la reconstrucción de la imagen del componente de lito localizado en la pelvis renal; volumen porcentual litiásico pélvico (VLP\%): porcentaje del volumen del lito a nivel de pelvis en relación con el volumen litiásico total; volumen litiásico cáliz de entrada (VLCE): volumen calculado del componente de lito en cáliz de entrada; volumen porcentual litiásico cáliz de entrada (VLCE\%): porcentaje del volumen del lito a nivel del cáliz de entrada en relación con el volumen litiásico total; longitud infundibular (LI): medido como la distancia entre el punto más distal del cáliz que contiene el lito y el punto medio del labio inferior de la pelvis renal; ancho infundibular (AI): medido como el punto más estrecho en el eje del infundíbulo inferior; ángulo intercalicial (AIC): determinado por la intersección del eje infundibular del cáliz de entrada (línea que conecta el centro de la pelvis con el fondo del cáliz de entrada) y el eje del cáliz deseado (línea que conecta el centro de la pelvis con un punto en el fondo del cáliz deseado); cáliz favorable (CF): cáliz con carga litiásica cuyo eje infundibular forma un ángulo obtuso (mayor de $90^{\circ}$ ) en relación con el cáliz de entrada, asociado con un ancho infundibular mayor de $8 \mathrm{~mm}$; cáliz no favorable (CNF): cáliz con carga litiásica cuyo eje infundibular forma un ángulo agudo (menor de $90^{\circ}$ ) en relación con el cáliz de entrada, asociado con un ancho infundibular menor de $8 \mathrm{~mm}$; volumen litiásico cáliz favorable (VLCF): volumen calculado del componente de lito en cáliz favorable; volumen litiásico cáliz no favorable (VLCNF): volumen 
calculado mediante reconstrucción de la imagen del componente de lito localizado en el cáliz no favorable; volumen porcentual litiásico cáliz favorable (VLCF\%): porcentaje del volumen del lito a nivel del cáliz favorable en relación con el volumen litiásico total; volumen porcentual litiásico cáliz no favorable (VLCNF\%): porcentaje del volumen del lito a nivel del cáliz no favorable en relación con el volumen litiásico total; estos parámetros se midieron antes y después de la operación y se clasificaron de acuerdo con la descripción de Mishra y colaboradores. Todas las mediciones las efectuó un urólogo para su análisis. Figura 1 y 2

Los pacientes se clasificaron según lo descrito por Mishra y colaboradores en tres tipos: Tipo 1: litos coraliformes con un volumen litiásico total menor de $5000 \mathrm{~mm}^{3}$ más volumen litiásico cáliz

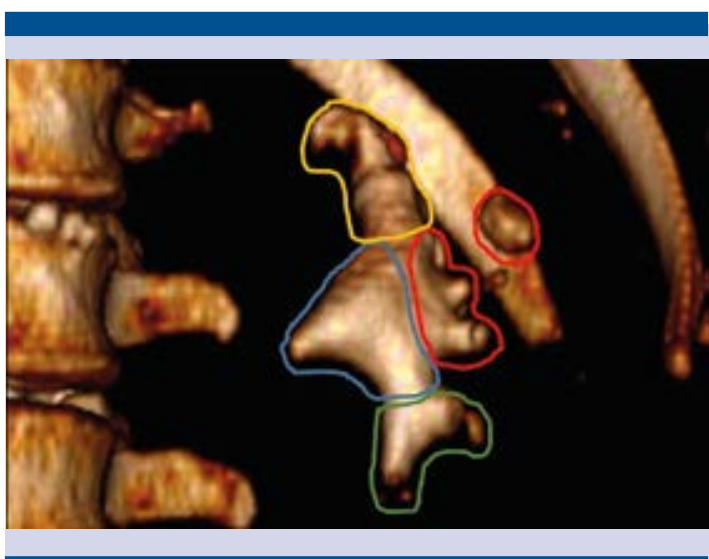

Figura 2. Reconstrucción volumétrica en un modelo de superficie en 3D de un lito coraliforme. La morfometría litiásica se demuestra de la siguiente manera; Azul=Volumen litiásico pélvico, Verde= Volumen litiásico cáliz de entrada, Naranja= Volumen litiásico cáliz favorable, Rojo=Volumen litiásico cáliz no favorable. ${ }^{11}$

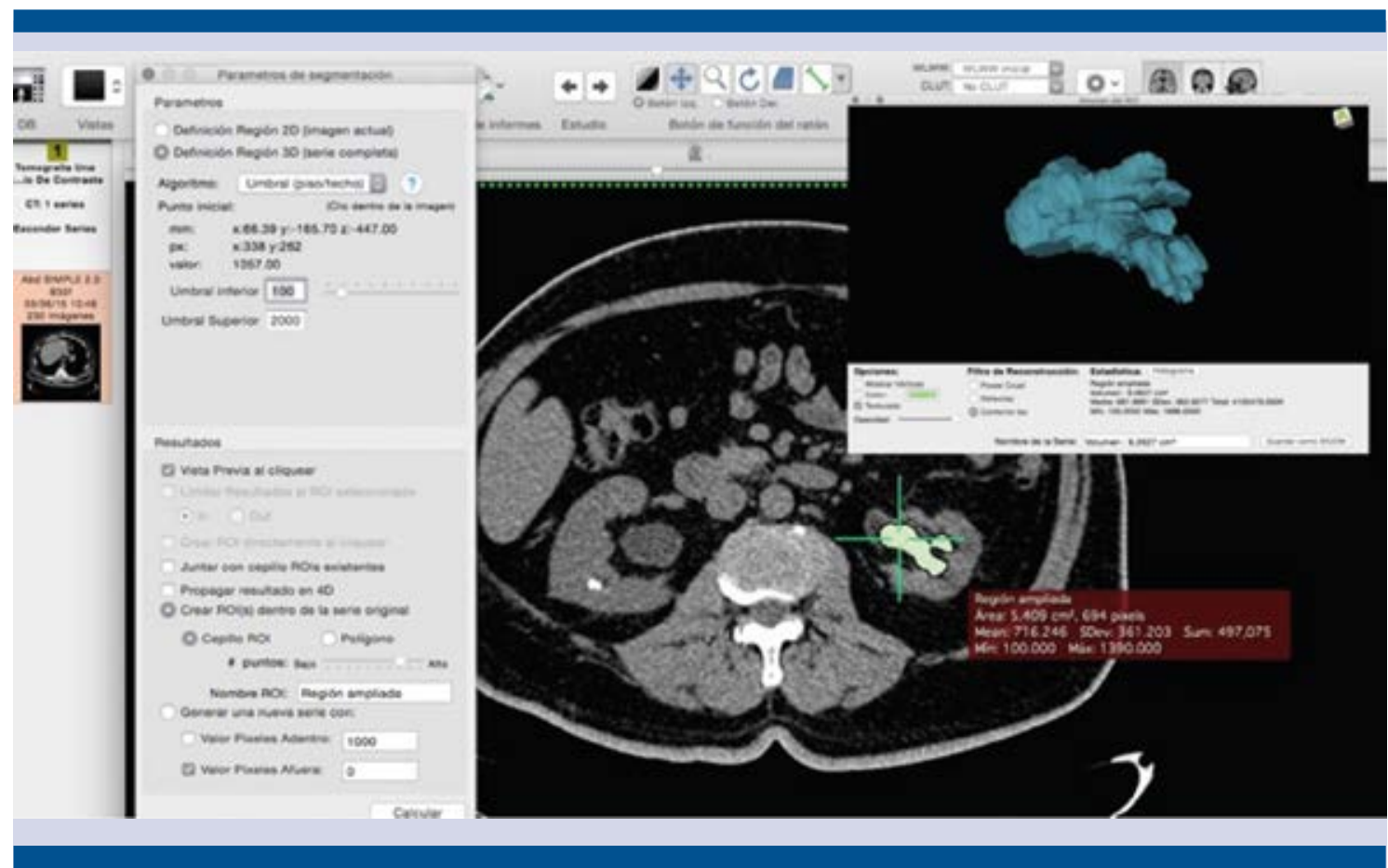

Figura 1. Programa visor de imágenes Osiri ${ }^{\circledR}$ con reconstrucción volumétrica de lito coraliforme. 
no favorable: menor de 5\%; Tipo 2: volumen litiásico total: 5000-20,000 mm³ más volumen litiásico cáliz no favorable: 5- 10\%; y el Tipo 3: volumen litiásico total mayor de $20,000 \mathrm{~mm}^{3}$ y un volumen litiásico cáliz no favorable mayor de $10 \%$. Figura 3

\section{Análisis de datos}

Se utilizó el paquete de análisis estadístico para la ciencia social versión 21.0 (SPSS, Inc, Chicago, Illinois, U.S.A.). Como medidas estadísticas descriptivas se usaron: tendencia central, media, mediana (mínimo - máximo) para las variables métricas y la frecuencia (porcentaje) para variables categóricas. Para la información continua se utilizó $t$ pareada y $\chi^{2}$ para la categórica. Los puntos de corte determinados se utilizaron según lo descrito por Mishra y colaboradores, en tres tipos: Tipo 1: litos coraliformes con un volumen litiásico menor de $5000 \mathrm{~mm}^{3}$ más volumen litiásico cáliz no favorable: menor de 5\%; Tipo 2: volumen litiásico: 5000-20 $000 \mathrm{~mm}^{3}$ más volumen litiásico cáliz no favorable: 5-10\%; y Tipo 3: volumen litiásico mayor de 20,000 $\mathrm{mm}^{3}$ y un volumen litiásico cáliz no favorable mayor

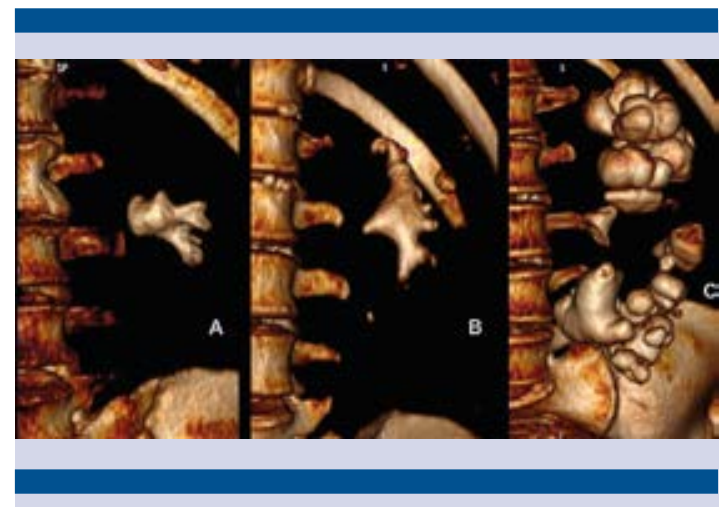

Figura 3. Clasificación volumétrica de la litiasis coraliforme, según descrita por Mishra y colaboradores. Tipo 1, lito coraliforme con un VLT: $<5000 \mathrm{~mm}^{3}+$ VLCNF: <5\% (A); Tipo 2, VLT: 5000-20 $000 \mathrm{~mm}^{3}+$ VLCNF: $5-10 \%$ (B); y el Tipo 3, VLT > $20000 \mathrm{~mm}^{3} \mathrm{y}$ un VLCNF $>10 \%(C)$. de $10 \%$, se construyeron modelos de predicción de riesgo relativo, con intervalos de confianza (IC) de 95\%. Se aceptó como estadísticamente significativo un valor de $\mathrm{p}<0.05$.

\section{RESULTADOS}

Se incluyeron 46 pacientes: 26 hombres (53.1\%) y 20 mujeres $(40.8 \%)$ con edad promedio de $49.5 \pm 12.18$ años (límites 17 y 78 años). De estos mismos pacientes se estudiaron 46 unidades renales, lo que correspondió a una frecuencia de 21 casos de lado derecho (42.9\%) y 25 casos de lado izquierdo (51.0\%). En cuanto al número de procedimientos y tractos se dividieron en tres grupos. Para un procedimiento se observó una frecuencia de 26 casos (53.1\%), dos procedimientos: 19 casos $(38.8 \%$ ) y tres procedimientos: 1 caso $(2.0 \%)$; para un tracto 31 casos $(63.3 \%)$, dos tractos: 13 casos $(26.5 \%)$ y tres tractos: 2 casos $(4.1 \%)$. En todos los casos se describió la localización del tracto de entrada, con una frecuencia de 31 casos (63.3\%) en el cáliz inferior, 9 casos (18.4\%) en el cáliz medio y 6 casos (12.2\%) en el cáliz superior. También se anotó la existencia de algún tipo de derivación urinaria, previo al procedimiento con nefrolitotomía percutánea, se observaron 20 casos (40.8\%) con uso de catéter ureteral tipo doble J. Cuadro 1

En el total de los casos se observó un eje longitudinal de $25.76 \pm 11.59 \mathrm{~mm}$ (límites 8 y $70 \mathrm{~mm}$ ), un eje trasversal de $15.11 \pm 8.85 \mathrm{~mm}$ (límites 6 y $40 \mathrm{~mm}$ ), carga litiásica de $350.74 \pm 376.92$ (límites 38 y 2198), densidad de $989.43 \pm 288.44 \mathrm{UH}$ (límites 500 y $1500 \mathrm{UH}$ ), un volumen litiásico total de $6597.74 \pm 11933.93 \mathrm{~mm}^{3}$ (límites 260 y $\left.75805 \mathrm{~mm}^{3}\right)$, un VLP de $1759.65 \pm 2573.45$ $\mathrm{mm}^{3}$ (límites 200 y $12200 \mathrm{~mm}^{3}$ ), un VLP\% 30.46 $\pm 26.40 \%$, un VLCE de $1472.54 \pm 4046.77 \mathrm{~mm}^{3}$ (límites 230 y $26885 \mathrm{~mm}^{3}$ ), un VLCE\% de 24.26 $\pm 25.10 \%$, una LI de $26.24 \pm 10.31 \mathrm{~mm}$ (límites 12 y $59 \mathrm{~mm}$ ), un Al de $9.91 \pm 3.58 \mathrm{~mm}$ (límites 2 y $25 \mathrm{~mm}$ ), un AIC de $105.93 \pm 23.48^{\circ}$ (límites 
Cuadro 1. Datos demográficos $(n=46)$

\begin{tabular}{|c|c|c|c|c|}
\hline $\begin{array}{l}\text { Factores de } \\
\text { riesgo }\end{array}$ & $\mathbf{n}$ & Media \pm DE & Rango & $\%$ \\
\hline Edad & 46 & $49.5 \pm 12.18$ & $17-78$ & \\
\hline \multicolumn{5}{|l|}{ Sexo } \\
\hline Masculino & 26 & & & 53.1 \\
\hline Femenino & 20 & & & 40.8 \\
\hline \multicolumn{5}{|l|}{ Lateralidad } \\
\hline Derecho & 21 & & & 42.9 \\
\hline Izquierdo & 25 & & & 51.0 \\
\hline \multicolumn{5}{|l|}{ Tamaño del lito } \\
\hline $\begin{array}{l}\text { Eje longitudi- } \\
\text { nal }(\mathrm{mm})\end{array}$ & & $25.76 \pm 11.59$ & $8-70$ & \\
\hline $\begin{array}{l}\text { Eje transverso } \\
(\mathrm{mm})\end{array}$ & & $15.11 \pm 6.85$ & $6-40$ & \\
\hline $\begin{array}{l}\text { Densidad del lito } \\
\text { (UH) }\end{array}$ & & $989.43 \pm 288.44$ & $500-1500$ & \\
\hline
\end{tabular}

$\mathrm{n}=$ Número de casos incluidos; $\mathrm{DE}=$ Desviación estándar; $\mathrm{UH}=$ Unidades Hounsfield .

60 y $\left.146^{\circ}\right)$, un VLCF de $1488.93 \pm 4150,95 \mathrm{~mm}^{3}$ (límites 450 y $27600 \mathrm{~mm}^{3}$ ), un VLCF\% de 23.87 $\pm 25.56 \%$, un VLCNF de $1177.74 \pm 5263.91$ $\mathrm{mm}^{3}$ (límites 210 y $35960 \mathrm{~mm}^{3}$ ), y VLCNF\% de $9.22 \pm 11.27 \%$.

Para la comparación de variables se dividió a la población en grupos según el número de tractos realizados, tracto único (Grupo I), dos tractos (Grupo II) y más de dos tractos (Grupo III), no existiendo diferencias entre estos en cuanto a edad, sexo o lateralidad. Se registró una preferencia por el cáliz inferior como cáliz de entrada en los tres grupos, la carga litiásica fue de menor grado en aquellos pacientes con un solo tracto en comparación aquellos con dos o más. Cuadro 2

En la comparación de la morfometría litiásica, el volumen litiásico total y el volumen litiásico del cáliz no favorable, se observo estadísticamente significativo en los tres grupos para determinación en cuanto al número de tractos $(p<0.001)$. El volumen litiásico del cáliz de entrada y el volumen litiásico de cáliz favorable se observó con mediciones de $1120 \pm 987 \mathrm{~mm}^{3}$ vs 17586 $\pm 13150 \mathrm{~mm}^{3}(\mathrm{p}<0.001), 1109 \pm 993 \mathrm{~mm}^{3}$ vs $17586 \pm 13150 \mathrm{~mm}^{3}(\mathrm{p}<0.001)$, al comparar el Grupo II vs Grupo III, mientras que el volumen litiásico porcentual de cáliz no favorable se observó en $8.55 \pm 10.68 \%$ vs $25.50 \pm 30.40 \%$ y $8.31 \pm 8.24 \%$ vs $25.50 \pm 30.40 \%$ (p 0.001 y p 0.010), al comparar al Grupo I vs Grupo III y Grupo II vs Grupo III para determinación del número de tractos, respectivamente. Cuadro 3

De acuerdo con los puntos de corte establecidos por Mishra y colaboradores se compararon los grupos para determinar el riesgo en cuanto al número de tractos o de procedimientos necesarios para obtener tasas libres de litiasis, siendo la división de estos en base a tracto y/o procedimiento único (Grupo I) vs dos o mas tractos y/o procedimientos (Grupo II).

Se observó que para el número de tractos necesarios para alcanzar tasas libres de litiasis el volumen litiásico total, el volumen porcentual litiásico del cáliz no favorable y el volumen litiásico del cáliz favorable, fueron estadísticamente significativas $(p<0.001, p<0.001$, $\mathrm{p}=0.059$, respectivamente). En contraparte para la valoración del número de procedimientos necesarios para los mismos puntos de corte, se advirtió que el volumen litiásico total y la carga litiásica, fueron estadísticamente significativos $(p=0.002$ y $p=0.011)$. Cuadro 4 y 5

\section{DISCUSIÓN}

La bibliografía internacional relacionada con el tratamiento de pacientes con litiasis recomienda la nefrolitotomía percutánea como opción de primera línea para el tratamiento intrarrenal de litos $\geq 2 \mathrm{~cm}$. Los conceptos de tracto (s) y procedimiento (s) son clínicamente relevantes para planear una nefrolitotomía percutánea como monoterapia para tratar pacientes con litiasis 
Sedano-Basilio JE y col. Morfometría litiásica

Cuadro 2. Correlación clínica del número de tractos para la nefrolitotomía percutánea como monoterapia.

\begin{tabular}{|c|c|c|c|}
\hline Variable & Tracto único & Dos Tractos & Mas de dos tractos \\
\hline Numero de unidades renales & 31 & 13 & 2 \\
\hline Edad (media $\pm D E$ ), años & $51.3 \pm 13.3$ & $45.4 \pm 8.1$ & $47.5 \pm 12.0$ \\
\hline Sexo $(H: M)$ & $19: 12$ & $5: 8$ & $2: 0$ \\
\hline Lateralidad (D:I) & $15: 16$ & $5: 8$ & $1: 1$ \\
\hline Cáliz de entrada (I:M:S) & $18: 7: 6$ & $11: 2: 0$ & $2: 0: 0$ \\
\hline Localización litos (I:M:S:Mu) & $9: 3: 1: 18$ & $0: 1: 0: 12$ & $0: 0: 0: 2$ \\
\hline Catéter JJ previo (Si:No) & $12: 19$ & $7: 6$ & $1: 1$ \\
\hline Eje longitudinal (mm) & $20.84 \pm 4.74$ & $31.85 \pm 10.51$ & $62.50 \pm 10.60$ \\
\hline Eje transverso (mm) & $12.26 \pm 4.01$ & $18.31 \pm 3.52$ & $38.50 \pm 2.12$ \\
\hline Carga litiásica $\left(\mathrm{mm}^{3}\right)^{*}$ & $206.65 \pm 94.08$ & $456.31 \pm 175.89$ & $1898.00 \pm 424.26$ \\
\hline Densidad (UH) & $1009.06 \pm 295.16$ & $972.54 \pm 291.69$ & $795.00 \pm 134.35$ \\
\hline \multicolumn{4}{|l|}{ Complicaciones } \\
\hline Clavien I & 27 & 9 & 2 \\
\hline Clavien II & 2 & 4 & 0 \\
\hline Clavien III & 2 & 0 & 0 \\
\hline Clavien IV & 0 & 0 & 0 \\
\hline
\end{tabular}

DE = Desviación estándar; H:M = Hombre : Mujer; D:I = Derecho : Izquierdo; I:M:S = Inferior : Medio : Superior; I:M:S:Mu = Inferior : Medio : Superior : Múltiples; UH = Unidades Hounsfield.

* Carga litiásica $=0.785 \times$ Longitud $_{\text {Máximo }} \times$ Ancho $_{\text {Máxim }}$

Cuadro 3. Correlación clínica de la morfometría litiásica con el número de tractos necesarios para la nefrolitotomía percutánea como monoterapia

\begin{tabular}{|c|c|c|c|c|c|c|}
\hline Variable & $\begin{array}{l}\text { Tracto único } \\
\qquad(\mathrm{n}=31)\end{array}$ & $\begin{array}{l}\text { Dos Tractos } \\
\qquad(n=13)\end{array}$ & $\begin{array}{c}\text { Mas de dos } \\
\text { tractos } \\
(n=2)\end{array}$ & $\begin{array}{l}\text { Único } \\
\text { Vs Dos** }\end{array}$ & $\begin{array}{c}\text { Dos } \\
\text { Vs Mas de } \\
\text { dos } * *\end{array}$ & $\begin{array}{c}\text { Único } \\
\text { Vs Mas de } \\
\text { dos** }\end{array}$ \\
\hline Volumen litiásico total $\left(\mathrm{mm}^{3}\right)^{*}$ & $2753 \pm 1423$ & $8031 \pm 2764$ & $56867 \pm 26781$ & 0.001 & $<0.001$ & $<0.001$ \\
\hline Volumen litiásico pélvico $\left(\mathrm{mm}^{3}\right)^{*}$ & $894 \pm 924$ & $2594 \pm 2547$ & $9742 \pm 3476$ & 0.002 & 0.624 & $<0.001$ \\
\hline Volumen \% litiásico pélvico & $30.77 \pm 28.65$ & $31.62 \pm 23.03$ & $17.50 \pm 2.12$ & 0.211 & 0.216 & 0.077 \\
\hline $\begin{array}{l}\text { Volumen litiásico cáliz de entrada } \\
\left(\mathrm{mm}^{3}\right)^{*}\end{array}$ & $580 \pm 474$ & $1120 \pm 987$ & $17586 \pm 13150$ & 0.043 & $<0.001$ & $<0.001$ \\
\hline $\begin{array}{l}\text { Volumen litiásico cáliz favorable } \\
\left(\mathrm{mm}^{3}\right)^{*}\end{array}$ & $589 \pm 569$ & $1109 \pm 993$ & $17900 \pm 13717$ & 0.139 & $<0.001$ & $<0.001$ \\
\hline Volumen \% litiásico cáliz favorable & $27.58 \pm 29.61$ & $14.23 \pm 10.49$ & $29.0 \pm 9.89$ & 0.006 & 0.915 & 0.241 \\
\hline $\begin{array}{l}\text { Volumen litiásico cáliz no favorable } \\
\left(\mathrm{mm}^{3}\right)^{*}\end{array}$ & $244 \pm 276$ & $689 \pm 607$ & $18820 \pm 24239$ & $<0,001$ & $<0,001$ & $<0,001$ \\
\hline $\begin{array}{l}\text { Volumen \% litiásico cáliz no } \\
\text { favorable }\end{array}$ & $8.55 \pm 10.68$ & $8.31 \pm 8.24$ & $25.50 \pm 30.40$ & 0.433 & 0.001 & 0.010 \\
\hline \multicolumn{7}{|l|}{ Procedimientos } \\
\hline Único & 23 & 3 & 0 & 0.705 & 0.060 & $<0.001$ \\
\hline Dos & 8 & 9 & 2 & 0.532 & 0.008 & $<0.001$ \\
\hline Mas de dos & 0 & 1 & 0 & 0.001 & 0.422 & NV \\
\hline
\end{tabular}

* Media \pm Desviación estándar.

**Valor de $p$ obtenido mediante prueba de $\chi^{2}$.

$\mathrm{n}=$ número de casos incluidos; $\mathrm{NV}=$ No valorable. 
Cuadro 4. Morfometría litiásica y otros predictores en relación al número de tractos basados en puntos de corte

\begin{tabular}{|c|c|c|c|}
\hline Variable & $\begin{array}{l}\text { Tracto único } \\
(\mathrm{n}=31)\end{array}$ & $\begin{array}{c}\text { Dos o mas Tractos } \\
(n=15)\end{array}$ & Valor de $\mathbf{P} *$ \\
\hline Volumen litiásico total $\left(\mathrm{mm}^{3}\right)$ & & & $<0.001$ \\
\hline$<5000$ & $3(9.67 \%)$ & $13(86.66 \%)$ & \\
\hline$>5000$ & $28(90.32 \%)$ & $2(13.33 \%)$ & \\
\hline Volumen litiásico cáliz no favorable (\%) & & & 0.493 \\
\hline$<10$ & $19(61.29 \%)$ & $10(66.66 \%)$ & \\
\hline$>10$ & $12(38.70 \%)$ & $5(33.33 \%)$ & \\
\hline Carga litiásica $\left(\mathrm{mm}^{3}\right)$ & & & $<0.001$ \\
\hline$<400$ & $31(100 \%)$ & $5(38.46 \%)$ & \\
\hline$>400$ & 0 & $10(61.63 \%)$ & \\
\hline Volumen litiásico cáliz de entrada $\left(\mathrm{mm}^{3}\right)$ & & & 0.188 \\
\hline$<1000$ & $24(77.41 \%)$ & $9(60.0 \%)$ & \\
\hline$>1000$ & $7(22.58 \%)$ & $6(40.0 \%)$ & \\
\hline \multicolumn{4}{|l|}{ Volumen litiásico cáliz favorable $\left(\mathrm{mm}^{3}\right)$} \\
\hline$<1000$ & $25(80.64 \%)$ & $8(53.33 \%)$ & 0.059 \\
\hline$>1000$ & $6(19.35 \%)$ & $7(46.66 \%)$ & \\
\hline
\end{tabular}

* Valor de $p$ obtenido mediante prueba de $\chi^{2}$.

$\mathrm{n}=$ número de casos incluidos.

Cuadro 5. Morfometría litiásica y otros predictores en relación al número de procedimientos basados en puntos de corte

\section{Variable}

Volumen litiásico total $\left(\mathrm{mm}^{3}\right)$

$\begin{array}{ccc}<5000 & 22(84.61 \%) & 8(40.0 \%) \\ >5000 & 4(15.38 \%) & 12(60.0 \%)\end{array}$

Volumen litiásico cáliz no favorable (\%)

$\begin{array}{ccc}<10 & 15(57.69 \%) & 14(70.0 \%) \\ >10 & 11(42.30 \%) & 6(30.0 \%)\end{array}$

Carga litiásica $\left(\mathrm{mm}^{3}\right)$

$\begin{array}{ccc}<400 & 24(92.30 \%) & 12(60.0 \%) \\ >400 & 2(7.69 \%) & 8(40.0 \%)\end{array}$

Volumen litiásico cáliz de entrada $\left(\mathrm{mm}^{3}\right)$

$\begin{array}{ccc}<1000 & 19(73.07 \%) & 14(70.0 \%) \\ >1000 & 7(26.92 \%) & 6(30.0 \%)\end{array}$

Volumen litiásico cáliz favorable $\left(\mathrm{mm}^{3}\right)$

$\begin{array}{ccc}<1000 & 21(80.76 \%) & 12(60.0 \%) \\ >1000 & 5(19.23 \%) & 8(40.0 \%)\end{array}$

* Valor de P obtenido mediante prueba de $\chi^{2}$. 
coraliforme. ${ }^{11} \mathrm{~A}$ pesar de que la cantidad de pérdida de la función renal se describe como similar entre tracto único vs múltiples, esta última opción se asocia con más complicaciones hemorrágicas. ${ }^{21-25}$

Las desventajas de la nefrolitotomía percutánea, realizada como monoterapia en múltiples procedimientos, son: mayor estancia intrahospitalaria, costos y complicaciones. Por esto es pertinente disminuir el número de tractos o procedimientos a lo mínimamente requerido, mientras se alcancen las tasas libre de litiasis más altas. ${ }^{11}$

La carga litiásica es un factor determinante en este tipo de procedimientos para alcanzar tasas libres de litiasis; sin embargo, en la actualidad aún no existen parámetros volumétricos debidamente definidos para valorar el número de tractos o procedimientos para el tratamiento exitoso de estos padecimientos.

Uno de los estudios pivote para determinar la morfometría litiásica coraliforme y su repercusión en el número de tractos o procedimientos necesarios a realizar, para obtener tasas libres de litiasis lo llevaron a cabo Mishra y su grupo en 2012, en el que mediante la medición volumétrica del total del lito coraliforme, y su distribución en el sistema colector, determinaron cómo estas se correlacionan con la nefrolitotomía percutánea para el número de tractos o procedimientos necesarios para obtener tasas libres de litiasis como monoterapia. En sus resultados describen que en el análisis univariado el volumen litiásico total $(p=0.013)$, volumen litiásico en cáliz no favorable ( $p=0.007)$, volumen litiásico porcentual pélvico $(p=0.026)$, volumen litiásico en cáliz de entrada y pelvis $(p<0.001)$ y un cáliz no favorable $(p=0.001)$ predijeron el número de tractos necesarios $(p<0.001)$, mientras que el volumen litiásico total $(p<0.001)$, el volumen litiásico pélvico ( $p=0.0046)$, el volumen litiásico en cáliz no favorable $(p<0.001)$, volumen litiásico porcentual pélvico ( $p=0.04)$, volumen litiásico en cáliz de entrada y pelvis $(p=0.046)$ predijeron el número de procedimientos. En el análisis multivariado se demostró que un volumen litiásico porcentual de cáliz no favorable predijo el número de tractos (área bajo la curva, [AUC] - 0.91), mientras que un volumen litiásico total y volumen litiásico porcentual de cáliz no favorable (AUC - 0.846) predijeron el número de procedimientos. En su estudio los modelos de predicción de riesgo sugirieron que los litos coraliformes con una distribución de Tipo I requerirá solo un procedimiento y un tracto (litos coraliformes con un volumen litiásico total: menores de $5000 \mathrm{~mm}^{3}$ con un volumen porcentual litiásico cáliz no favorable menor de 5\%), el Tipo 2 requerirá un tracto y uno o más procedimientos, o múltiples tractos en un procedimiento (litos coraliformes con un volumen litiásico total: 5000-20,000 $\mathrm{mm}^{3}$ con un volumen porcentual litiásico de cáliz no favorable de 5-10\%), y el Tipo 3, por tanto, requerirá de múltiples tractos y múltiples procedimientos (volumen litiásico total mayor de $20,000 \mathrm{~mm}^{3}$ y un volumen litiásico porcentual en cáliz no favorable mayor de $10 \%)$. Entre sus conclusiones mencionan que su modelo predice el número de tractos y procedimientos para la nefrolitotomía percutánea como monoterapia, y que la morfometría litiásica coraliforme logra diferenciar los cálculos en Tipo 1 (tracto y procedimiento, único); Tipo 2 (tracto único-múltiples procedimientos o tractos múltiples- procedimiento único) y Tipo 3 (tracto y procedimientos múltiples). ${ }^{11,20}$

En la actualidad hay publicaciones que ya describen el uso de estas variables como predictoras de nefrolitotomía percutánea como monoterapia en pacientes con litiasis coraliforme, Ganpule y su grupo, en 2012, promovieron el uso de este nuevo sistema de clasificación para predecir el número de tractos o procedimientos para el tratamiento de estas entidades, que ayudan al cirujano a planear el procedimiento, abordaje 
y la concesión de información al paciente antes de la cirugía. ${ }^{20}$

En nuestro estudio en comparación a los anteriormente mencionados, observamos que un volumen litiásico total mayor de $5,000 \mathrm{~mm}^{3}$ tiene un riesgo de 8.25 y 60.6 veces mayor para dos o más procedimientos o tractos, necesarios para obtener una tasa libre de litiasis (IC95\%: 2.05-33.16 y 9.01-40.16), respectivamente. $Y$ basados en estos hallazgos, las características volumétricas pueden ser consideradas para normar conductas en el tratamiento de la litiasis coraliforme mediante nefrolitotomía percutánea.

Una de las limitaciones de este ensayo es que es retrospectivo y necesita continuar su seguimiento de forma prospectiva para validar sus resultados. Otra limitante fueron los puntos de corte descritos en las publicaciones de Mishra y colaboradores, y que en nuestra población no se observó como estadísticamente significativo el volumen litiásico porcentual del cáliz no favorable para la determinación del número de tractos ni el número de procedimientos.

\section{CONCLUSIONES}

La morfometría litiásica es una clasificación clínica con un concepto interesante para la nefrolitotomía percutánea como monoterapia. La distribución intrarrenal y las proporciones volumétricas propias del lito son características que pueden ser de ayuda para determinar el número de tractos o procedimientos necesarios para alcanzar tasas libres de litiasis. Se requieren estudios prospectivos que tomen en cuenta variables como: estancia hospitalaria, complicaciones trans y postoperatorias y los costos. La interfaz de usuario con el programa visor de imágenes es intuitiva, guía al especialista de forma natural durante la interpretación de imágenes y determinación de las características y proporciona un plan preoperatorio más fidedigno para el cirujano. De los elementos tomados en cuenta en nuestro estudio, el volumen litiásico total representó el factor de mayor riesgo para el número de tractos o procedimientos necesarios para alcanzar tasas libres de litiasis en pacientes con litos coraliformes a quienes se realizó nefrolitotomía percutánea, en nuestra población.

Nota

Los autores no recibieron ningún patrocinio para llevar a cabo este estudio/articulo por parte de ninguna organización publica o privada.

El Dr. Carlos Martínez Arroyo pertenece al grupo de consultoría por parte de la marca BARD.

El resto de los autores previamente mencionados declaramos que no existe ningún tipo de conflicto de interés.

Se agradece por su participación y apoyo a todos los integrantes de este trabajo.

\section{REFERENCIAS}

1. Clark JY, Thompson IM, Optenberg SA. Economic impact of urolithiasis in the United States. J Urol 1995;154 (6):2020-2024.

2. Scott R. Epidemiology of stone disease. $\mathrm{Br} J$ Urol 1985;(57):491-497.

3. Curhan C, Rimm E, Willet W, Stampfer M. Regional variation in nephrolithiasis incidence and prevalence among United States men. J Urol 1994;(151):838-841.

4. Danpure Ch. Genetic disorders and urolithiasis. Ur Clin North Am 2000;27(2):287-299.

5. Tiselius HG. Epidemiology and medical management of Stone disease. BJU Int 2003;(91):758-767.

6. Turk C, Knoll T, Petrik A, et al. EAU Guidelines on Urolithiasis. European Association of Urology, 2016.

7. Ritter M, Krombach P, Michel M. Percutaneous Stone removal. European Urology Supplements 2011;10:433-439.

8. Barradas E, Guzmán F, Cortez R. Experiencia inicial en nefrolitotomía percutánea en el Centro Médico Nacional "20 de Noviembre". Rev Mex Urol 2008;68(2):69-87.

9. Usawachintachit M, Masic S, Allen IE, et al. Adopting Ultrasound Guidance for Prone Percutaneous Nephrolithotomy: 
Evaluating the Learning Curve for the Experienced Surgeon. J Endourol. 2016 Aug; 30(8):856-63.

10. Ibarluzea G, Gamarra M, Gallego JA, et al. Litotricia renal percutánea. Evolución, indicaciones y metodología actual en nuestra unidad de litotricia. Arch. Esp. de Urol. 2001; 54 (9): 951-969.

11. Mishra S, Sabnis R, Desai M. Staghorn morphometry: a new tool for clinical classification and prediction model for percutaneous nephrolithotomy monotherapy. Journal of Endourology 2012;26 (1):6-14.

12. Thiruchelvam N, Mostafid H, Ubhayakar G. Planning percutaneous nephrolithotomy using multidetector computed tomography urography, multiplanar reconstruction and three-dimensional reformatting. BJU International 2005;95:1280-1284.

13. Mishra S, Sabnis R, Desai M. Percutaneous nephrolithotomy monotherapy for staghorn: paradigm shift for 'staghorn morphometry' based clinical classification. Curr Opin Urol 2012; 22 (2): 148-153.

14. Smith A, Averch T, Shahrour K, et al. A nephrolithometric nomogram to predict treatment success of percutaneous nephrolithotomy. The Journal of Urology July 2013;190:149-153.

15. Labadie K, Okhunov Z, Akhavein A, et al. Evaluation and comparison of urolithiasis scoring systems used in percutaneous kidney stone surgery. J Urol. 2015 Jan;193(1):154-9.

16. Cho HJ, Lee JY, Kim SW, et al: Percutaneous nephrolithotomy for complex renal calculi: is multitract approach ok? Can J Urol 2012;19: 6360
17. El-Tabey NA, El-Nahas AR, Eraky I, et al. Long-term functional outcome of percutaneous nephrolithotomy in solitary kidney. Urology 2014; 83:1011.

18. Wood K, Keys T, Mufarrij P et al: Impact of Stone removal on renal function: a review. Rev Urol 2011;13:73.

19. Gorbachinsky I, Wood K, Colaco M, et al. Evaluation of Renal Function after Percutaneous Nephrolithotomy-Does the Number of Percutaneous Access Tracts Matter? J Urol. 2016 Jul; 196 (1): 131-6.

20. Ganpule A, Mahesh R. What's new in percutaneous nephrolithotomy. Arab Journal of Urology 2012; 10: 317-323.

21. Hegarty NJ, Desai MM. Percutaneous nephrolithotomy requiring multiple tracts: Comparison of morbidity with single-tract procedures. J Endourol 2006;20:753-760.

22. Akman T, Sari E, BinbayM, et al.Comparison of outcomes after percutaneous nephrolithotomy of staghorn calculi in those with single and multiple accesses. J Endourol 2010;24:955-960.

23. Aron $M$, Yadav R, Goel R, et al. Multi-tract percutaneous nephrolithotomy for large complete staghorn calculi. Urol Int 2005;75:327-332.

24. Handa RK, Evan AP, Willis LR, et al. Renal functional effects of multiple-tract percutaneous access. J Endourol 2009;23: 1951-1956.

25. Kukreja R, Desai M, Patel S, et al. Factors affecting blood loss during percutaneous nephrolithotomy: Prospective study. J Endourol 2004;18:715-722.

\section{AVISO PARA LOS AUTORES}

Revista Mexicana de Urología tiene una nueva plataforma de gestión para envío de artículos. En: https://www.revisionporpares.com/index.php/RMUrol podrá inscribirse en nuestra base de datos administrada por el sistema Open Journal System (OJS) que ofrece las siguientes ventajas para los autores:

- Subir sus artículos directamente al sistema.

- Conocer, en cualquier momento, el estado de los artículos enviados, es decir, si ya fueron asignados a un revisor, aceptados con o sin cambios, o rechazados.

- Participar en el proceso editorial corrigiendo y modificando sus artículos hasta su aceptación final. 